\title{
Potentiale und Herausforderungen neuer Vermessungstechnologien im Bergbau - Der Einsatz unbemannter Flugkörper zur Erfassung von Geodaten
}

\author{
Alexander Tscharf \\ Lehrstuhl für Bergbaukunde, Bergtechnik und Bergwirtschaft, Department Mineral Resources Engineering, \\ Montanuniversität Leoben, Leoben, Österreich
}

Eingegangen 11. August 2016; angenommen 12. August 2016; online publiziert 9. September 2016

\begin{abstract}
Zusammenfassung: Die bergmännische Vermessung ist ein wichtiger Teil der Mineralrohstoffgewinnung. Sie hat die Aufgabe und das Ziel, in allen Phasen des Mineralrohstoffprojekts, beginnend von der Erkundung über die Erschließung und Gewinnung, bis hin zur Nachsorge die notwendigen raumbezogenen Daten zu generieren. Durch den Einsatz moderner UAV-gestützter photogrammetrischer Systeme (UAV: unmanned aerial vehicle) in Kombination mit einer leistungsfähigen und automatisierten Mehrbildauswertung können zukünftig und gegenwärtig viele Vermessungsaufgaben schneller, kostengünstiger und sicherer durchgeführt werden. Im vorliegenden Beitrag werden Anwendungspotentiale unbemannter Luftfahrzeuge in der Mineralrohstoffindustrie aufgezeigt, die UAVVermessung - vor allem vor dem Hintergrund der Ergebnisgüte - den gängigen Verfahren gegenübergestellt und Nachteile diskutiert.
\end{abstract}

Schlüsselwörter: Photogrammetrie, Unbemannte Flugsysteme, Bildbasierte 3D Rekonstruktion, Genauigkeitsanalyse, UAV-Vermessung im Bergbau

Potentials and Challenges of New Surveying Technologies in Mining - The Use of Unmanned Aerial Vehicles for Geospatial Data Acquisition

Abstract: Mine surveying is an important part of mining science and technology. It includes all measurements, calculations and mapping which serve the purpose of ascertaining and documenting geospatial information at all stages

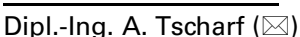

Lehrstuhl für Bergbaukunde, Bergtechnik und Bergwirtschaft, Department Mineral Resources Engineering,

Montanuniversität Leoben,

Franz-Josef-Str. 18,

8700 Leoben, Österreich

alexander.tscharf@unileoben.ac.at
}

from prospecting to exploitation and utilizing mineral deposits. Using unmanned aerial vehicles (UAVs) in conjunction with automated multi-view processing pipelines offers new ways for aerial data acquisition to perform surveying tasks in a more flexible, faster, cheaper and safer way. In this paper the potential of unmanned aircraft in the mineral resource industry is shown, especially in the light of accuracy, UAV-surveying is compared to conventional methods and disadvantages are discussed.

Keywords: Photogrammetry, Unmanned aerial system, Image-based 3D reconstruction, Accuracy evaluation, UAV-surveying in mining

\section{Einleitung}

Die rasanten technologischen Entwicklungen im Bereich der unbemannten Luftfahrzeuge (unmanned aerial vehicle, UAV), führen zu einem vermehrten Einsatz dieser Technologie in den unterschiedlichsten Anwendungsbereichen und rücken vergleichbare Systeme überdies immer stärker in den Fokus der Öffentlichkeit [1]. Gemeinsam mit dem Aufkommen dieser modernen Trägerplattformen erlebt auch die Photogrammetrie bzw. die bildbasierte 3D Rekonstruktion eine technologische Renaissance und bildbasierte, ursprünglich aus dem Bereich der Computervisualistik stammende, Verfahren finden zusehends Eingang in die vermessungstechnische Forschung, Entwicklung und Anwendung.

Auch in der bergmännischen Vermessung kommen vergleichbare Systeme, vor allem zur Nachtragung des Bergbaukartenwerks [2], zur Massenermittlung, sowie zur Erhebung des Ist-Zustandes als Planungsgrundlage immer häufiger zum Einsatz. Diese Systeme vereinen die Vorteile der luftgebundenen und der terrestrischen Photogrammetrie, sind in der Lage, nahezu jeden Aufnahmepunkt im Raum 
Abb. 1: Kategorisierung gem. LBTH 67 (nach [8]). Abhängig von Einsatzgebiet und Betriebsmasse müssen unterschiedliche Anforderungen erfüllt werden, um eine Bewilligung zum Einsatz des Flugsystems zu erhalten

\begin{tabular}{|l|c|c|c|c|}
\cline { 2 - 5 } \multicolumn{1}{c|}{} & \multicolumn{4}{c|}{ Einsatzgebiet } \\
\cline { 2 - 5 } & $\begin{array}{c}\text { I } \\
\text { unbebaut }\end{array}$ & $\begin{array}{c}\text { II } \\
\text { unbesiedelt }\end{array}$ & $\begin{array}{c}\text { III } \\
\text { besiedelt }\end{array}$ & \begin{tabular}{c} 
IV dicht besiedelt \\
\hline Betriebsmasse bis einschließlich 5kg
\end{tabular} \\
\cline { 2 - 5 } & A & A & B & C \\
\hline Betriebsmasse bis einschließlich 25kg & A & B & C & D \\
\hline Betriebsmasse über 25kg und bis & B & C & D & D \\
\hline
\end{tabular}

einzunehmen und können so typische geometrische Einschränkungen, insbesondere im Tagebau, umgehen [3, 4].

Im Folgenden werden nach einer kurzen Einführung in die theoretischen Grundlagen die technischen Möglichkeiten dieser neuen Verfahren vor allem in Hinblick auf relevante Anwendungsfälle präsentiert. Ergänzend wird auch der in Österreich derzeit gültige rechtliche Rahmen zum Betrieb unbemannter Luftfahrzeuge thematisiert, wobei jedoch das Hauptaugenmerk auf die in der Mineralrohstoffindustrie relevanten Teile und Einsatzgebiete gelegt wird. Es wird auch versucht, die UAV Vermessung den gängigen Methoden (GNSS und Tachymetrie sowie bemannte Luftbildphotogrammetrie) gegenüberzustellen, und Potentiale wie auch systembedingte Schwächen aufgezeigt.

\section{Stand der Technik}

Unbemannte Flugsysteme haben ihren Ursprung in militärischen Anwendungen, wobei die ersten zivilen Nutzungen Przybilla und Wester-Ebbinghaus [5] zugeschrieben werden. Hervorgerufen durch den technologischen Fortschritt im Bereich des Flugzeugbaus, der Navigationstechnik und der Mechatronik unterliegt die bildbasierte UAV-Vermessung einer stetigen Weiterentwicklung, sowohl im Bereich der eingesetzten Flugsysteme als auch der nachgeschalteten Auswertung [1,6]. Durch den „Einstieg“ der Vermessungsbranche in die laufenden Entwicklungen tritt vor allem die Frage nach der Genauigkeit bzw. der Qualität der Ergebnisse immer stärker in den Vordergrund, was auch den aktuellen Schwerpunkt der gegenständlichen Forschung an der Montanuniversität darstellt.

\subsection{Rechtslage}

Wie in mittlerweile fast allen europäischen Ländern wurde auch in Österreich auf den vermehrten Einsatz unbemannter Flugsysteme reagiert und das Luftfahrtgesetz [7] mit 01.01.2014 novelliert, wodurch sich grundlegende Änderungen für die zivile Nutzung der unbemannten Luftfahrt und somit auch für den Einsatz unbemannter Flugkörper zur Vermessung im Bergbau ergaben. Auf Basis dieser Novelle ist der Betrieb unbemannter Flugkörper zu Vermessungszwecken nun möglich, wobei die technischen und betrieblichen Voraussetzungen berücksichtigt werden müssen. Jedes Flugsystem bedarf der Bewilligung durch die Austro
Control, wobei die Bewilligung in Bescheidform auf Antrag des Bewilligungswerbers erfolgt.

Nahezu alle zur Vermessung eingesetzten Systeme sind der sogenannten Klasse 1 zugeordnet, wobei diese Systeme nur mit direkter - und ohne technische Hilfsmittel bestehender - Sichtverbindung zum Piloten betrieben werden dürfen und hinsichtlich der Genehmigung unter den Geltungsbereich der LBTH 67 (Lufttüchtigkeits- und Betriebstüchtigkeitsanforderungen für unbemannte Luftfahrzeuge bis $150 \mathrm{~kg}$ [8]) fallen. Abhängig von Betriebsmasse und Einsatzgebiet werden diese noch weiter kategorisiert (Abb. 1), was wiederum unterschiedliche Luftüchtigkeits- und Betriebstüchtigkeitsanforderungen im Bewilligungsverfahren zur Folge hat - wie zum Beispiel redundante Energie- und Datenversorgung oder spezielle Ausbildungskriterien für Piloten. Während die technische Zulassung der Kategorien A und B noch eher einer Modellbauzulassung entspricht, findet in den höheren Kategorien eine strenge technische Überprüfung des Gerätes und all seiner ausfallsgefährdeten Komponenten statt [7, 8].

Unabhängig von der jeweiligen Kategorie dürfen Luftfahrzeuge der Klasse 1 allerdings nur bis zu einer Höhe von $150 \mathrm{~m}$ und innerhalb des österreichischen Bundesgebiets betrieben werden. Höhere Flüge beziehungsweise Umstände, unter denen mit einem Überfliegen der Bundesgrenzen gerechnet werden muss, bedürfen einer gesonderten $\mathrm{Be}$ willigung durch die Austro Control $[7,8]$.

Zusätzlich zu den luftfahrtrechtlichen Bestimmungen müssen selbstverständlich auch alle übrigen rechtlichen Bestimmungen berücksichtigt werden, wobei hier insbesondere das Datenschutzgesetz und der allgemeine zivilrechtliche Schutz der Privatsphäre, Rechte der Grundeigentümer bei Start und Landung, das Versicherungsrecht sowie auch fernmelderechtliche Regularien zu nennen sind.

\subsection{Photogrammetrische Grundlagen}

Wie bereits erwähnt, erlebt gemeinsam mit dem vermehrten Einsatz unbemannter Flugsysteme auch die Photogrammetrie einen Aufschwung in der vermessungstechnischen Forschung und Anwendung. Hervorgerufen durch die Flexiblität müssen bei der Aufnahme mittels UAV ähnlich der terrestrischen Nahbereichsphotogrammetrie zumeist beliebige Bildkonfigurationen ausgewertet werden, wobei die Bestimmung der relativen bzw. äußeren Orientierung (räumliche Lage und Orientierung der Bilder zum Zeitpunkt der Bildaufnahme) einen der wesentlichsten 
Abb. 2: Vollautomatische Mehrbildauswertungen sind in der Lage, aus einer Vielzahl an ungeordneten Bildern die äußere Orientierung und eine punktbasierte Szenenrekonstruktion zu errechnen (nach [10])

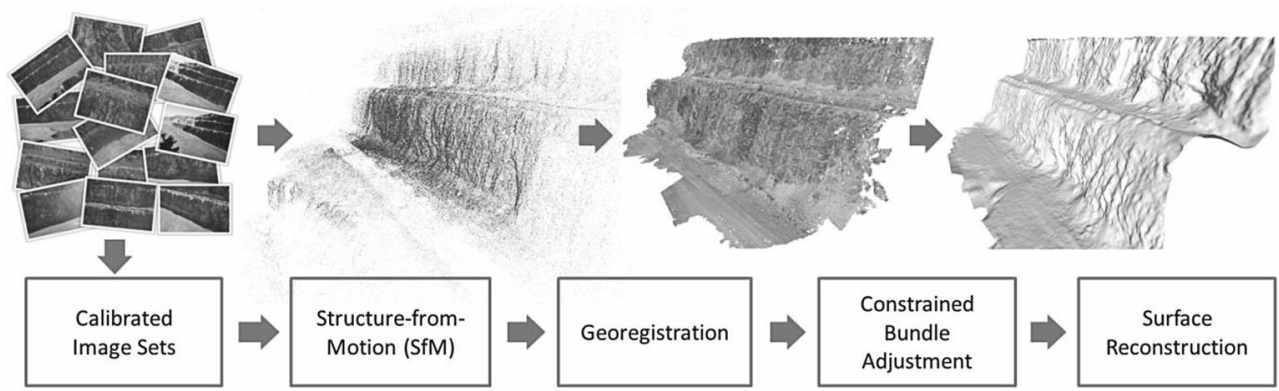

Schritte darstellt. Obwohl nahezu alle eingesetzten Systeme über entsprechende on-board Sensorik (GNSS, IMU) zur Bestimmung der Plattform- bzw. Sensororientierung verfügen, können nicht zuletzt aufgrund der notwendigen Miniaturisierung zumeist keine ausreichenden Genauigkeiten erreicht werden. Mit dem Aufkommen vollautomatischer, bildgestützter 3D Rekonstruktionen [9] wurde jedoch die notwendige Schätzung der äußeren Orientierung signifikant vereinfacht und die Auswertung der oftmals unregelmäßigen Bildverbände einer UAV-Befliegung erst ermöglicht (Abb. 2).

\subsubsection{Structure from Motion}

Die angesprochenen Verfahren (Structure from Motion) sind in der Lage, aus einer Vielzahl an (zunächst ungeordneten) Bildern die äußere Orientierung der Kamera und eine punktbasierte Szenenrekonstruktion zu errechnen. Die einzige Voraussetzung ist ein ausreichender Überlapp zwischen den Bildern, die überdies ausreichend Textur zeigen müssen, um die Korrespondenzanalyse zwischen den unterschiedlichen Aufnahmen zu ermöglichen.

Je nach Anwendung können die so erhaltenen Ergebnisse in weiterer Folge noch verdichtet beziehungsweise zu digitalen Oberflächenmodellen vermascht werden. Abb. 2 zeigt schematisch den Ablauf der beschriebenen Auswertung, wobei im Folgenden noch kurz auf die wesentlichsten Schritte der Auswertung eingegangen wird:

Kamerakalibrierung: Bestimmung der inneren Orientierung (Fehllage des Bildhauptpunkts, fokale Länge und Verzeichnungsparameter); basierend auf dem Modell der Lochkamera (Zentralprojektion).

Merkmalsextraktion und Korrespondenzanalyse: Finden von Features und Berechnung der Korrespondenzen basierend auf der euklidischen Distanz zwischen den Deskriptoren im Eigenschaftsraum [11].

Belative Orientierung und Bündelblockausgleich: Schätzung der relativen Lage der Bilder zueinander und anschließende simultane Optimierung der inneren und der äußeren Orientierung, sowie der 3D-Objektpunkte [12].

\subsubsection{Georeferenzierung}

Aufgrund der fehlenden Längenmessung - rein bildbasierte Verfahren beruhen ausschließlich auf der Messung von Winkeln - befinden sich die, durch das bis hierhin beschriebene Verfahren generierten, Ergebnisse zunächst nur in einem lokalen euklidischen Koordinatensystem, welches durch eines der gefundenen Kamerapaare festgelegt wird. Eine metrische Skalierung der Punktwolke kann durch die Integration zumindest einer bekannten Distanz in die Auswertung erreicht werden, was wiederum durch einen in der Szene und in der Rekonstruktion eindeutig zuzuordnenden Maßstab, wie zum Beispiel einen bekannten Abstand zwischen zwei Kamerapositionen oder die Distanz zwischen zwei markanten Punkten erfolgen kann. Für vermessungstechnische Anwendungen ist jedoch die absolute Position der Objektpunkte von wesentlicher Bedeutung, wozu das Modell mittels räumlicher Ähnlichkeitstransformation (3D-Helmert Transformation, 7-Parameter Transformation, beziehungsweise "Similarity Transform“) unter der Verwendung von zumindest 3 Vollpasspunkten vom Modellsystem in das übergeordnete Referenzsystem (System der Landesvermessung) transformiert wird [13].

Bei rein bildbasierter Rekonstruktion ist zu beobachten, dass der Fehler zu den Rändern hin zunimmt. Es kommt zu einer Verformung des Bildblocks im Bündelblockausgleich, der einerseits auf eine schlechte Kamerakalibrierung und dadurch künstlich hervorgerufene Fehlerbeiträge in der Optimierung, und andererseits auf den in den Randbereichen geringeren Bildüberlapp zurückzuführen ist. Eine mittlerweile in nahezu allen kommerziellen Auswerteprodukten implementierte Möglichkeit, die durch Verformung des Bildblocks entstehenden systematischen Fehler zu vermeiden, ist die Einbringung bekannter Pass- und Kontrollpunkte sowie der grob per Onboard-GPS ermittelten Bildaufnahmepositionen in den Bündelblockausgleich (engl. bundle adjustment). Dies führt einerseits zu kleineren Residuen und andererseits lässt sich ein gleichzeitiger Übergang in ein entsprechendes Referenzkoordinatensystem bewerkstelligen [10].

\section{UAV-gestützte Photogrammetrische Vermessung}

Die regelmäßige Vermessung des Betriebes ist zur Nachtragung des Bergbaukartenwerks ( $\$ 42$ Markscheideverordnung 2013), zur Massenermittlung sowie auch zur Qualitätssteuerung unbedingt erforderlich. Aufgrund der immer schneller fortschreitenden Betriebe, vor allem im Tagebau, und aufgrund des hohen personellen und zeitlichen Aufwands bei der konventionellen Vermessung werden Abbaustätten teilweise nur unregelmäßig und in großen 


\begin{tabular}{|ll}
\hline \multicolumn{2}{|l}{$\begin{array}{l}\text { TABELLE } 1 \\
\text { Gegenüberstellung Flächenflugzeug mit }\end{array}$} \\
\hline Flächenflugzeuge & Multirotorsysteme \\
\hline Vorteile & \\
\hline Vermessung größerer Areale in kürzerer Zeit & Flexible Aufnahmegeometrie (Detailaufnahmen möglich) \\
\hline Längere Flugdauer möglich (> 45 min) & Möglichkeit, die Position zu halten und zu schweben \\
\hline $\begin{array}{l}\text { I.d.R. größere Flughöhen } \rightarrow \text { geringere Datenmen- } \\
\text { gen }\end{array}$ & Geringer Platzbedarf für Start und Landung \\
\hline- & $\begin{array}{l}\text { Größere Lasten können getragen werden } \rightarrow \text { größeres Einsatzspektrum für } \\
\text { unterschiedliche Sensorik }\end{array}$ \\
\hline Nachteile & \\
\hline $\begin{array}{l}\text { Zumeist sind nur vertikale Aufnahmen möglich } \\
\text { (Sichtschatten) }\end{array}$ & Kürzere Flugdauer (10-15 min) $\rightarrow$ geringere Reichweite \\
\hline „Start- und Landebahn“ notwendig & Vermessung ganzer Abbaustätten nur mit mehreren Starts möglich \\
\hline I.d.R. größere Flughöhen $\rightarrow$ geringere Auflösung & - \\
\hline
\end{tabular}

Zeitintervallen vermessen. Während bemannte Luftbildphotogrammetrie für die in Österreich üblichen kleineren Betriebsstätten oftmals aus Kostengründen keine wirkliche Alternative darstellt, ist bei terrestrischen Verfahren die Standortwahl zumeist stark von der Objektgeometrie beeinflusst. Kleine und leicht zu steuernde unbemannte Flugsysteme sind nun in der Lage, die Lücke zwischen luftgebundenen und terrestrischen Verfahren zu schließen, wodurch sich viele für die Mineralrohstoffgewinnung relevante Anwendungspotentiale auftun, die im Folgenden dargestellt werden sollen.

\subsection{Flugplattformen}

Gemeinsam mit der stetig wachsenden Anzahl an Herstellern nimmt auch die Anzahl der unterschiedlichen Fluggerätetypen zu, wobei die Erhöhung der Sicherheit und die Vergrößerung des Einsatzspektrums durch Hybridformen als Hauptentwicklungsrichtung ausgemacht werden können.

Nach wie vor am häufigsten im Einsatz stehen jedoch leichte Flächenflugzeuge und Multirotorsysteme, die mit ihren Vor- und Nachteilen (vor dem Hintergrund des Einsatzes im Bergbau) in Tab. 1 gegenübergestellt werden.

\subsection{Anwendungspotentiale}

Durch die neu gewonnene Perspektive ergeben sich speziell für die in Österreich üblichen kleineren Gewinnungsstätten breite Anwendungsfelder und vor allem eine einfache und kostengünstige Alternative zu bemannter Luftbildphotogrammetrie.

Neben der direkten Verwendung der Bilder zur fotografischen Dokumentation bzw. zur Inspektion kritischer Bereiche stellen vor allem hochaufgelöste, tagesaktuelle und „richtige“" (vertikale Flächen wie Fassaden dürfen nicht erkennbar sein) Orthofotos eine wichtige Datengrundlage für die Rohstoffgewinnung dar. Im Gegensatz zu einer "herkömmlichen“ Luftbildaufnahme gibt ein Orthofoto wie eine maßstäbliche Karte - ein verzerrungsfreies und maßstabsgetreues Bild der Erdoberfläche, das basierend auf einem digitalen Geländemodell von der ursprünglichen Zentralprojektion in eine Parallelprojektion umgerechnet wurde. Abgesehen von der Möglichkeit, jederzeit aktuelles Bildmaterial aufnehmen zu können, unterscheiden sich aus UAV-Befliegungen abgeleitete Orthofotos vor allem durch die Qualität des zugrundeliegenden Geländemodells von online verfügbaren Bildern aus Geodatenportalen. Ein hoch aufgelöstes Geländemodell ist unbedingte Voraussetzung für die richtige Umbildung der Luftbilder zu einem Orthofoto, da nur jene Objekte, die auch im Geländemodell erfasst wurden, bei der Orthorektifizierung berücksichtigt werden können [14].

Die hochaufgelösten digitalen Geländemodelle (Bodenauflösung abhängig von der Flughöhe im Bereich weniger Zentimeter), die mittels Structure from Motion weitestgehend automatisiert aus den aufgenommenen Bildern abgeleitet werden, dienen jedoch nicht nur als Grundlage für Orthofotos, sondern liefern auch für sich wertvolle Information über die beflogene Gewinnungsstätte. Insbesondere bei der Verwendung von Multirotorsystemen ist eine lückenlose, dreidimensionale Erfassung des gesamten Gebiets und sogar die Modellierung überhängender Bereiche aus sicherer Entfernung möglich. Die absolute Genauigkeit dieser Modelle beläuft sich bei entsprechender und sorgfältig geplanter Vorgehensweise auf unter $10 \mathrm{~cm}$, womit auch die gesetzlichen Vorgaben bei der Tagebauvermessung [2] eingehalten werden können.

Naturgemäß ist auch eine weitere Verwendung dieser 3D Information zur Ableitung von lückenlosen Schnittdarstellungen und Profilen sowie zur Volums- und Massenermittlung möglich. Durch die einfache und flexible Möglichkeit der Datengewinnung wird eine vollständige Überwachung der Materialbewegungen ermöglicht.

\subsection{Systembedingte Schwächen}

Der vorliegende Beitrag soll jedoch in keiner Weise als „Werbung" für den Einsatz von UAVs verstanden werden, weshalb neben den dargestellten Möglichkeiten an dieser Stelle auch auf systembedingte Schwachpunkte eingegangen wird. 
Abb. 3: Geringe Fluggeschwindigkeit, hoher Bildüberlapp und eine an das Objekt angepasste Blickrichtung der Kamera führen zu den besten Ergebnissen [15]
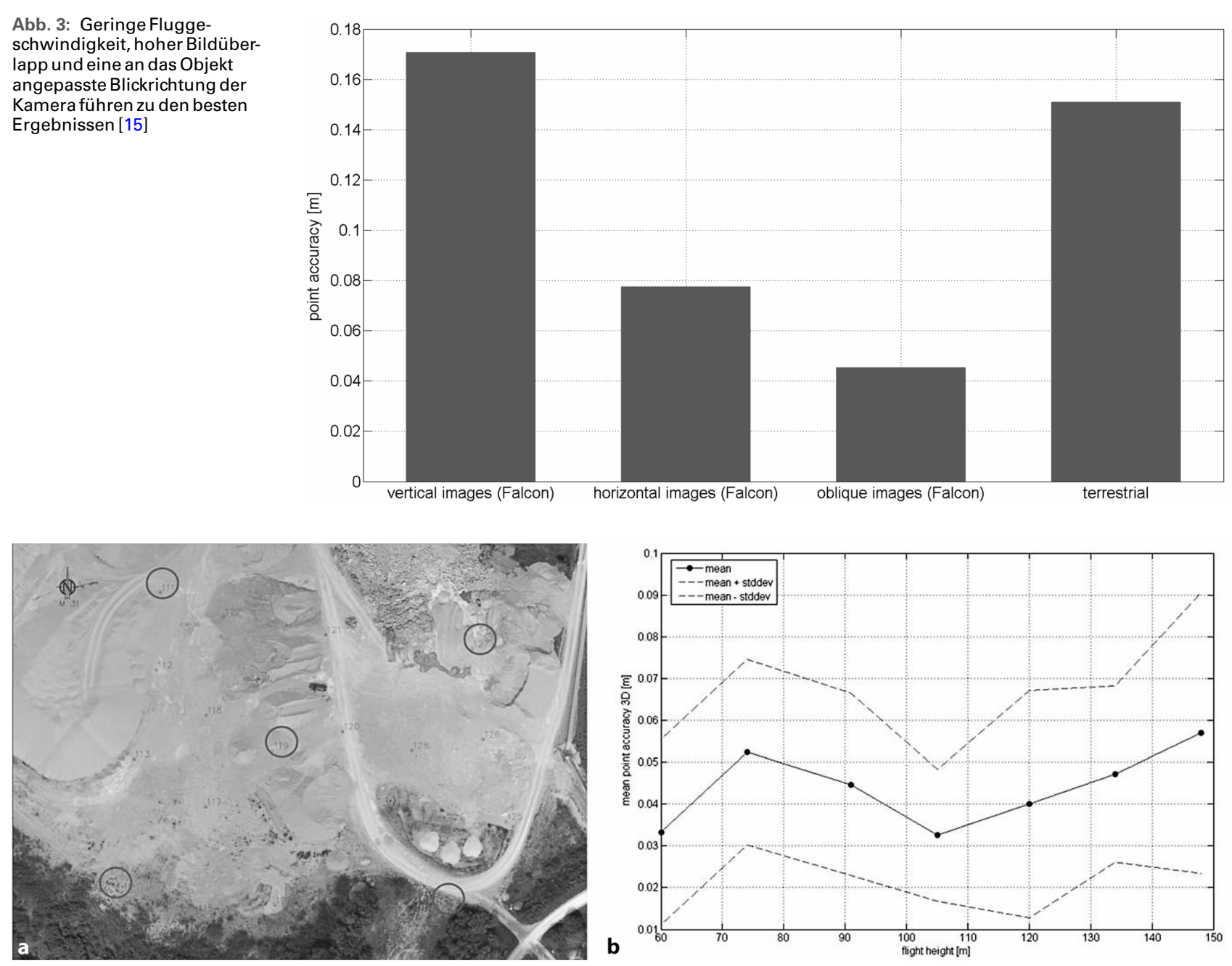

Abb. 4: a Fünf(mit Kreisenmarkiert)derinsgesamt 19 Punktewurden als Passpunkte für die Schätzung derTransformationsparameterim Zuge der Georeferenzierung verwendet. b Die Gegenüberstellung der mittleren Fehler mit der Flughöhe zeigt eine leichte Zunahme der Fehler mit steigender Höhe, wobei alle Fehler im einstelligen Zentimeterbereich liegen. (nach [16])

Während der zeitliche Aufwand zur Vermessung im Feld durch die weitestgehend autonome Datenerfassung reduziert wird, stellt die weitere Verarbeitung der Daten sowie die Integration in gewohnte Arbeitsabläufe (Erzeugung gängiger Produkte aus neuen Datenquellen) eine nicht zu unterschätzende Herausforderung dar. Die Berechnungszeit für die Prozessierung der Bildverbände kann, abhängig von der vorhandenen Computerinfrastruktur und der Größe der Datensätze, mehr als $24 \mathrm{~h}$ in Anspruch nehmen, und überdies müssen die anfallenden Datenmengen (rund 30 bis $70 \mathrm{MB}$ pro Bild) gespeichert und gewartet werden.

Eine größere Herausforderung als die photogrammetrische Rekonstruktion und Speicherung der Daten ergibt sich jedoch aus der weiteren Nutzung der gewonnenen Information bzw. aus der Bearbeitung der Daten. In der vermessungstechnischen Anwendung übliche CAD-Systeme sind zur Bearbeitung von Massendaten nur bedingt geeignet und die Fülle an gewonnener Information ist auch nicht für alle Anwendungsfälle sinnvoll bzw. erforderlich. Bei der Verwendung herkömmlicher Methoden (Tachymeter, GNSS) erfolgt eine selektive Datenerfassung der re- levanten Information im Feld, während bei unbemannten Flugsystemen zunächst alles erfasst und die Interpretation und etwaige Reduktion der Daten zur Gänze in den Innendienst verlagert wird. Dies reduziert zwar das Risiko, wichtige Informationen zu übersehen, allerdings ist die Ableitung der tatsächlichen Gegebenheiten aus der getrennten Betrachtung der vorhandenen Geometrie- und Bildinformation nicht immer eindeutig möglich bzw. zumindest nach derzeitigem Stand der Technik nicht vollständig und nur zum Teil automatisierbar.

\subsection{Genauigkeit}

Vor allem vor dem Hintergrund der Ergebnisverantwortlichkeit gewinnt die Frage nach der tatsächlich erreichten bzw. erreichbaren Genauigkeit zusehends an Bedeutung. Theoretische Überlegungen sowie praktische Versuchsergebnisse zeigen deutlich, dass ein unkontrolliertes und völlig freies Fotografieren trotz hoher Redundanz nicht zwingend zu einer hohen Genauigkeit der 3D Objektpunkte führt. 
Abb. 5: Gegenüberstellung der UAV-Vermessung mitherkömmlichen Methoden. UAVs können konventionelle Methoden ergänzen, allerdings können nichtalle im Bergbau anfalrmessungsaufgaben mit ausreichender Qualität erfüllt werden

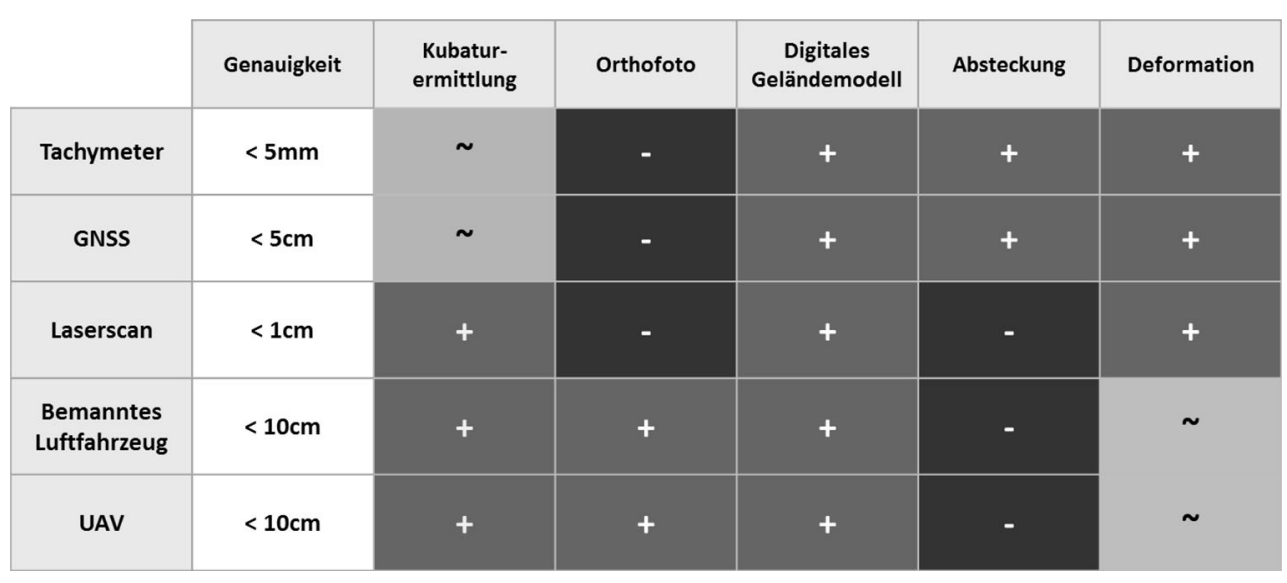

Zur Identifizierung und Quantifizierung der genauigkeitsrelevanten Einflussparameter wurden in den letzten Jahren mehrere Befliegungen mit verschiedenen Plattformen in unterschiedlichen Gewinnungsstätten durchgeführt. Die Evaluierung erfolgte als Vergleich zu einem Referenzmodell, welches über ein dichtes Netz an sowohl in den Bildern, als auch in der Örtlichkeit eindeutig identifizierbaren Referenzpunkten realisiert wurde. Zumeist wurden hierzu individuell identifizierbare „fiducial marker" eingesetzt und nur teilweise kamen rote, nichtcodierte Kreistargets zum Einsatz.

Im Zuge der Untersuchungen konnte gezeigt werden, dass durch den Einsatz flexibler UAVs ein signifikanter Genauigkeitszuwachs im Vergleich zu terrestrischer und bemannter Aero-Photogrammetrie erreicht werden kann (vgl. Abb. 3 am Beispiel einer Bruchwand am Steirischen Erzberg). Durch die optimale Anpassung der dreh- und schwenkbaren Kamera an die Objektgeometrie kann eine gleichmäßige Bildabdeckung des Objekts erreicht werden, und außerdem wirkt sich die Kombination unterschiedlicher Bildabstände und Auflösungen positiv auf die Stabilität des Bildverbandes aus.

Außerdem konnte anhand mehrerer Kampagnen gezeigt werden, dass die gesetzlichen Anforderungen der Markscheideverordnung 2013 [2] eingehalten werden können, wobei im Folgenden die Ergebnisse einer Kampagne aus dem Mai 2015 näher dargestellt werden. Im Rahmen der Kampagne wurden insgesamt 7 Flüge in unterschiedlichen Höhen durchgeführt. (von $60 \mathrm{~m}$ bis $150 \mathrm{~m}$ in $15 \mathrm{~m}$ Schritten), und zur Evaluierung der Genauigkeit wurden im 4,5 ha großen Gebiet insgesamt 19 Punkte durch Auslegen von Target-Tafeln bzw. durch Aufsprühen von Farbkreisen signalisiert und mittels Trimble S6 Totalstation hochgenau vermessen. Fünf gleichmäßig im Gebiet verteilte Punkte (in Abb. 4a mit Kreisen markiert) wurden als Passpunkte zur Georeferenzierung verwendet und die übrigen Punkte dienten als "Check Points" im Sinne der Genauigkeit. Als Sensorplattform wurde das Flächenflugzeug senseFly eBee, ausgerüstet mit einer Sony DSC-WX220 Kompaktkamera verwendet. Die Kamera hat eine Auflösung von $18 \mathrm{Mp}$, und es ist ein $1 / 2,3^{\prime \prime}$ Zoll CMOS Sensor mit einer physischen Pixelgröße von 1,3 $\mu \mathrm{m}$ verbaut. Durch die Bauart des Flugzeugs waren alle aufgenommenen Bilder
Vertikalaufnahmen, wobei sowohl Longitudinal- als auch Lateralüberlapp konstant bei 85 beziehungsweise $70 \%$ gehalten wurden.

In Abb. 4b kann deutlich erkannt werden, dass sowohl die mittleren Fehler als auch die Standardabweichungen ( $\pm \sigma$ Bereich als Visualisierung der Unsicherheit, angedeutet durch die gestrichelten Linien) mit steigender Flughöhe leicht zunehmen. Obwohl die mittleren Fehler bei allen untersuchten Flügen im einstelligen Zentimeterbereich liegen, deuten die verhältnismäßig großen Werte der Standardabweichung auf eine starke Streuung innerhalb der detektierten Fehler im Untersuchungsgebiet hin.

In allen durchgeführten Untersuchungen auffallend ist vor allem auch, dass bei Verwendung der beschriebenen Ausrüstung die höchste Genauigkeit bei einer Flughöhe von rund $100 \mathrm{~m}$ erreicht werden konnte. Die Begründung hierfür liegt vermutlich in einem Optimum aus Erkennbarkeit der natürlichen Features und Texturen sowie der Passpunkte und den dementsprechend genauen Bildmessungen, was in weiterer Folge zu einem gut stabilisierten Bildverband und einer hohen Genauigkeit führt [16].

Zusätzlich zu den Einflüssen, die sich aus der Bildaufnahme ergeben, muss selbstverständlich auch die Genauigkeit der konventionellen Passpunkteinmessung in die Betrachtungen miteinbezogen werden. Als Richtwert sollten die GCPs (Ground Control Points) mit 0,5* GSD (Ground Sample Distance) eingemessen werden. Bei einer in Österreich gesetzlich zulässigen Flughöhe von 150 m ergibt sich bei Verwendung der beschriebenen senseFly eBee eine Bodenauflösung von rund $4,5 \mathrm{~cm}$, was in weiterer Folge eine Passpunktgenauigkeit von $\pm 2 \mathrm{~cm}$ bedeutet. Diese Genauigkeitsanforderung kann jedoch mit moderner RTK- (Real Time Kinematic) GNSS- (Global Navigation Satellite System) Vermessung in der Regel eingehalten werden.

\section{Zusammenfassung und Ausblick}

Die Photogrammetrie, als flächiges und berührungsloses Verfahren, ist in der Lage, die Szene mit sehr hohem Detailgrad zu erfassen und vor allem in Kombination mit einem UAV können anfallende Vermessungsaufgaben schnell, kostengünstig und sicher durchgeführt werden. Aufgrund 
der geringen Betriebskosten im Vergleich zu bemannten Flugkörpern ist eine Befliegung auch bei in Österreich und Mitteleuropa üblichen, kleineren Betriebsstätten bereits wirtschaftlich, wodurch ein permanent aktuelles und vollständiges Kartenwerk bzw. auch eine vollständige Erfassung der bewegten Massen möglich ist.

Die Kehrseite der sehr schnellen Datenerfassung ist mit Sicherheit die aufwendigere, weil zeit- und vor allem rechenintensive, Auswertung. Eine zusätzliche Herausforderung ergibt sich außerdem in der weiteren Verarbeitung der Daten. Unbemannte Flugsysteme geben ein tagesaktuelles und vollständiges Bild ohne Interpretation und Filterung bei der Aufnahme im Feld. Die Interpretation bzw. Reduktion der Information wird zur Gänze in den Innendienst verlagert, was vor allem vor dem Hintergrund der Richtigkeit und Belastbarkeit der abgeleiteten Unterlagen von gewichtiger Bedeutung ist.

UAVs können somit herkömmliche Vermessungen zwar weitreichend unterstützen, aber keineswegs vollständig ersetzen. Mit Kameras ausgerüstete unbemannte Flugsysteme stellen eine wertvolle zusätzliche Informationsquelle dar, dienen jedoch grundsätzlich nur der Erfassung von Geodaten. Absteckungen sowie die Übertragung von Planungen in die Örtlichkeit sind, wie auch mit bemannten Luftfahrzeugen und Laserscannern, nicht möglich. (vgl. Abb. 5)

Hervorgerufen durch einen zunehmend stärkeren Wettbewerb unter den Herstellern und Anbietern werden die rasanten Entwicklungen der letzten Jahre wohl auch in den nächsten Jahren weiter anhalten. Aus anwendungsorientierter Sicht besteht der größte Forschungsbedarf mit Sicherheit im Bereich der Datenverarbeitung. Insbesondere durch die Kombination von semantischer und geometrischer Information könnte die Interpretation der Daten erleichtert bzw. automatisiert werden, wodurch die praktische Relevanz der bildgestützten UAV-Vermessung weiter gesteigert würde.

Open access funding provided by Montanuniversität Leoben.

Open Access Dieser Artikel wird unter der Creative Commons Namensnennung 4.0 International Lizenz (http://creativecommons.org/licenses/by/4.0/deed.de) veröffentlicht, welche die Nutzung, Vervielfältigung, Bearbeitung, Verbreitung und Wiedergabe in jeglichem Medium und Format erlaubt, sofern Sie den/die ursprünglichen Autor(en) und die Quelle ordnungsgemäß nennen, einen Link zur Creative Commons Lizenz beifügen und angeben, ob Änderungen vorgenommen wurden.

\section{Literatur}

1. Briese, C.; Fortner, R.; Sager, P.; Pfeifer, N.: Vom Modellflughobby zu unbemannten Flugsystemen für die Geodatenerfassung, VGI
Österreichische Zeitschrift für Vermessung und Geoinformation 101 (2013), Nr. 2+3, S. 64-74

2. Verordnung des Bundesministers für Wirtschaft, Familie und Jugend über Vermessungen beim Bergbau, das Bergbaukartenwerk und die Erfassung von Bodenbewegungen 2013 (Markscheideverordnung 2013), BGBI. II Nr. 437/2012

3. Rumpler, M.; Hoppe, C.; Wendel, A.; Mayer, G.; Bischof, H.: EchtzeitQualitätsüberprüfung für zuverlässige UAV-gestützte Bilddatenerfassung und exakte, automatisierte Mehrbildauswertung, VGI Österreichische Zeitschrift für Vermessung und Geoinformation 101 (2013), Nr. 2+3, S. 88-100

4. Mayer, G.; Pilgram, R.: Neue Entwicklungen in der Tagebauvermessung. In: Sroka, A. (Hrsg.): Schriftenreihe des Instituts für Markscheidewesen und Geodäsie, (2013-1), Freiberg: 2013, S. 36-48.

5. Przybilla, H.; Wester-Ebbinghaus, W.: Bildflug mit ferngelenktem Kleinflugzeug, Bildmessung und Luftbildwesen 47 (1979), S. $137-142$

6. Colomina I.; Molina, P.: Unmanned aerial systems for photogrammetry and remote sensing: A review, in: ISPRS Journal of Photogrammetry and Remote Sensing 92 (2014), S. 79-97

7. Bundesgesetz vom 2. Dezember 1957 über die Luftfahrt (Luftfahrtgesetz - LFG), BGBI. Nr. 253/1957, i.d.g.F.

8. Austro Control GmbH: Lufttüchtigkeits- und Betriebstüchtigkeitsanforderungen für unbemannte Luftfahrzeuge der Klasse 1 (2015), Lufttüchtigkeits- und Betriebstüchtigkeitshinweis Nr. 67 (LBTH 67), 2015

9. Hartley, R.; Zisserman, A.: Multiple View Geometry in Computer Vision, 2.ed., Cambridge University Press, 2003

10. Rumpler, M.; Daftry, S.; Tscharf, A.; Prettenthaler, R.; Hoppe, C.; Mayer, G.; Bischof, H.: Automated End-to-End Workflow for Precise and Geo-accurate Reconstructions Using Fiducial Markers. In: Photogrammetric Computer Vision - PCV 2014, 2014, pp. 135-142

11. Lowe, D.: Distinctive Image Features from Scale-Invariant Keypoints, International Journal of Computer Vision 60 (2004), iss. 2, pp. 91-110

12. Triggs, B.; McLauchlan, P.; Hartley, R.; Fitzgibbon, A.: Bundle adjustment - A modern synthesis, in: Zisserman, A.; Szeliski, R. (Hrsg.): Vision Algorithms: Theory and Practice, Berlin, Heidelberg: Springer, 2000, pp. 298-375

13. Lobnig, K.; Tscharf, A.; Mayer, G.: Einfluss der Georeferenzierung auf die absolute Rekonstruktionsgenauigkeit der photogrammetrischen UAV-gestützten Tagebauvermessung, in: BHM Berg- und Hüttenmännische Monatshefte, 160 (2015), H. 8, S. 373-378

14. Zurhorst, A.; Zurhorst, M.: Einsatz UAV-basierter Fotogrammetrie für Liegenschaftsvermessung, in: Internationale Geodätische Woche, Obergurgl 2015, Wichmann, 2015

15. Tscharf, A.; Rumpler, M.; Fraundorfer, F.; Mayer, G.; Bischof, H.: On the use of UAVs in mining and archaeology - geo-accurate 3D reconstructions using various platforms and terrestrial Views, in: ISPRS Annals for Photogrammetry, Remote Sensing and Spatial Information. Science, Volume II-1/W1, International Conference on Unmanned Aerial Vehicles in Geomatics, 30 Aug-02 Sep 2015, Toronto, Canada, 2015, pp 15-22

16. Tscharf, A.: Genauigkeitsevaluierung - Untersuchungen zur Genauigkeit der UAV-gestützten photogrammetrischen Vermessung im Bergbau, in Zusammenarbeit mit Friedl ZT GmbH, Interner Projektbericht zu FFG-Bridge "UAV Vermessung im Bergbau“ (841298), 2016, unveröffentlicht 